\title{
Evaluation of the effect of an "Internet plus medical"-based health management service model in patients with nonalcoholic fatty liver disease
}

Yan Yang ( $\sim$ yangyan1021@tom.com )

Shandong University Qilu Hospital https://orcid.org/0000-0001-5329-8451

Cui-Huan Tian

Shandong University Qilu Hospital

Jun Dai

Shandong University Qilu Hospital

Wei Zhang

Shandong University Qilu Hospital

Shu-Qing Li

Shandong University Qilu Hospital

Xiang Min

Shandong University Qilu Hospital

Yan-Yan Wang

Shandong University Qilu Hospital

Juan Cao

Shandong University Qilu Hospital

\section{Research article}

Keywords: Nonalcoholic fatty liver disease, health education, health management, quality of life

Posted Date: May 26th, 2020

DOI: https://doi.org/10.21203/rs.3.rs-15691/v3

License: (c) (i) This work is licensed under a Creative Commons Attribution 4.0 International License.

Read Full License 


\section{Abstract}

Objectives: To observe the effect of an "Internet plus medical"-based health management service model in patients with nonalcoholic fatty liver disease (NAFLD) and to explore an effective model for NAFLD health management to provide a reference for NAFLD treatment and nursing.

Methods: The present study was a randomized, controlled, parallel-group comparison trial. A total of 519 patients with NAFLD were randomly assigned to a routine health education group $(\mathrm{N}=258)$ or a health management platform group $(\mathrm{N}=261)$. The routine health education group received routine health education, and the health management platform group was treated with the "Internet plus medical"-based health management service model to manage NAFLD. The new model provides closed-loop services for the prevention and rehabilitation of NAFLD through the process of grouping, filing, evaluating, planning, intervening, assessing stage and following up. The two groups were observed for 24 weeks. The results of basic indicators, laboratory indicators, body composition analyses, controlled attenuation parameters (CAP) and quality of life assessment questionnaires were used as evaluation indices. All data of the participants were collected and analyzed prior to and following the intervention, and the differences between the two groups were compared.

Results: Compared with the routine health education group, the NAFLD health management service model based on "Internet plus medical" treatment effectively reduced the weight $(-3.80 \pm 3.11 \mathrm{vs}$ $-0.12 \pm 3.42, P=0.047)$, body mass index (BMI) $(-1.73 \pm 1.20$ vs $-0.14 \pm 1.68, P=0.031)$, CAP $(-48.42 \pm 10.13$ vs $4.13 \pm 7.45, P=0.044)$, aspartate transaminase (AST)/alanine aminotransferase (ALT) value $(0.18 \pm 0.37$ vs $0.04 \pm 0.11, P=0.037)$, body fat content $(-2.24 \pm 2.58$ vs $-0.86 \pm 2.78, P=0.194)$ and visceral fat area $(-10.87 \pm 15.34$ vs $-0.55 \pm 19.13, P=0.047)$ of NAFLD patients and improved the quality of life of NAFLD patients in terms of physiological role $(96.59 \pm 11.31$ vs $90.69 \pm 13.49, P=0.027)$, vitality ( $86.57 \pm 6.07$ vs $81.94 \pm 5.78, P=0.001)$ and general health $(78.05 \pm 6.35 v s 73.61 \pm 10.12, P=0.044)$.

Conclusions: The results of the present study preliminarily confirm the validity and development prospects of the "Internet plus medical"-based health management service model in NAFLD. This finding suggests that the model is an evidence-based model of health education and management for NAFLD prevention in China.

\section{Introduction}

Nonalcoholic fatty liver disease (NAFLD) is a disease in which excessive fat accumulates in the liver in the form of triglycerides, which is not caused by excessive alcohol intake. The pathological scope of NAFLD ranges from simple steatosis to nonalcoholic steatohepatitis (NASH), which can eventually develop into cirrhosis and is widely recognized as a global public health problem [1]. Worldwide, the incidence of NAFLD in the general population ranges from 28.01/1000 people every year to 52.34/1000 people every year [2]. In China, due to a large number of lifestyle and dietary changes, the prevalence of NAFLD is now as high as $20 \%-30 \%$, affecting a quarter of the total population $[3,4]$. Moreover, this 
situation is exacerbated by the increase in childhood obesity and the aging population, which are associated with some of the main causes of chronic liver disease worldwide. Without timely treatment, NAFLD can result in serious complications such as cirrhosis, hepatocellular carcinoma and even death [5, 6]. In addition to these liver complications, epidemiological studies have also demonstrated an increased risk of diabetes, metabolic syndrome and cardiovascular disease in NAFLD patients [7-16]. The increasing prevalence of NAFLD has led to a significantly increased healthcare and economic burden, so effective management and prevention is necessary.

NAFLD patients have poor self-management and compliance. The progression of NAFLD to nonalcoholic steatohepatitis (NASH) cirrhosis is very slow, but if NAFLD progresses to NASH, the risk of cirrhosis, liver failure and hepatocellular carcinoma will be greatly increased. According to the literature, in 10 years, the incidence of cirrhosis in NASH patients ranged from $15 \%$ to $25 \%$, and the mortality rate was approximately $30 \%$ to $40 \%$ [17-18]. At least $20 \%$ to $25 \%$ of NAFLD patients had body mass index (BMI), blood lipids, blood sugar and blood pressure in the normal range, namely, the so-called cryptogenic fatty liver. Compared with patients with chronic viral hepatitis, patients with NAFLD are much less concerned about their liver disease. Most NAFLD patients do not perceive NAFLD as a serious health threat and have poor treatment compliance. Because the pathogenesis of NAFLD is complex, there is no definite therapeutic drug to treat NAFLD. Both pioglitazone and vitamin E can improve steatohepatitis in NASH patients confirmed by biopsy. However, the long-term efficacy and safety of these two drugs are poorly demonstrated [19]. Therefore, lifestyle change is the cornerstone of treatment. At present, the only proven effective method to treat NAFLD is to reduce weight by optimizing lifestyle through diet and exercise [20]. However, it is difficult for patients to adhere to this method. Patients generally do not attach importance to the therapeutic value of lifestyle change. Therefore, health education for individuals with NAFLD is very important. It is necessary to strengthen health management education for patients to make them pay attention to their diseases, change their lifestyle and promote rehabilitation.

By promoting health management to change people's lifestyles, it has been shown that the mortality rate of cardiovascular diseases and diabetes mellitus in China has been reduced [21-22]. Moreover, the cost of healthcare can be effectively controlled by investing in health management. However, the management system for the prevention and treatment of NAFLD in China is not perfect and follows a single model of health management. Most health management systems are carried out by medical and health administrative departments, public health management institutes and community health service institutions. There is still a lack of continuity in management, which cannot meet the normal health needs of patients. Moreover, the low rate of patient follow-up has affected the operation of the continuous and full-process management system, so it is difficult to meet the real requirements of NAFLD patients. The use of innovative and digital media-based health education can be effective in improving health-based behavior such as physical activity [23]. By using Internet technology, associated medical teams form contractual cooperation with patients [24]. Responsible doctors explain the rights and obligations of both doctors and patients to patients in detail, explain their reasons and necessity in detail, restrain the behavior of patients, facilitate changes in their lifestyle, follow up regularly to monitor their implementation, and provide education and correction for nonimplementation of doctor's orders $[25,26]$. 
This system can improve the awareness and control rates of diseases. Thus, a dynamic cycle of followup assessment, guidance and health management is gradually formed. In this study, a novel health management service model based on "Internet plus medical" care for NAFLD was established and used to manage patients. Accordingly, the aim of this paper was to evaluate the role of this new model in improving the quality of life and health status of NAFLD patients and to explore an effective model of NAFLD prevention and control to improve the prevention and treatment of NAFLD.

\section{Materials And Methods}

\subsection{Ethics statement}

Permission was obtained from the Ethics Committee of Qilu Hospital of Shandong University, China. The date of approval by the ethics committee was 2018-02-12. Given that the centers were governmental, participation in our study was completely voluntary, and patients provided written informed consent.

\subsection{Design and participants}

\subsubsection{Sample sources}

People who participated in a NAFLD screening and physical examination at the health management center, Qilu Hospital of Shandong University from March 2018 to June 2018 with complete information were recruited.

The inclusion criteria for the study were as follows:

$\otimes$ Age $18-55$ years.

(2) NAFLD diagnosis based on two of the following three abdominal ultrasonographic findings:

QThe near-field echo of the liver was diffuse and stronger than that of the kidney.

$\unrhd$ The structure of the intrahepatic duct was not clearly displayed.

$\otimes$ The far-field echo of the liver gradually attenuated.

(3) Clear consciousness and ability to answer questions correctly and act freely.

(4) Use of a smart phone.

(5) No other Internet-based health management use.

(6) Voluntary participation in this study and provision of informed consent.

The exclusion criteria for the study were as follows: 
(1) Alcohol consumption history or equivalent alcohol intake of $>140 \mathrm{~g} /$ week for men and $>70 \mathrm{~g} / \mathrm{week}$ for women;

(2) Viral hepatitis, drug-induced liver disease, total parenteral nutrition, hepatolenticular degeneration, hemochromatosis, autoimmune liver disease, primary sclerosing cholangitis, primary biliary cirrhosis and other specific diseases that can cause fatty liver.

(3) Pregnant, lactating and reproduction-aged women who lack effective birth control.

(4) Patients with unstable angina pectoris, myocardial infarction, stroke or any serious adverse event within the first three months of the study.

(5) The use of any treatment that may affect liver function.

(6) Infectious diseases such as active pulmonary tuberculosis, AIDS or cancer.

(7) Diabetic individuals using insulin or hypoglycemic drugs.

(8) Physical disability, mental illness or an inability to provide written informed consent.

(9) Over the past three months or currently taking weight loss drugs or undergoing surgical treatment.

Termination or discontinuation of study:

(1) Participants who cannot attend follow-up or cooperate with the platform management and those lost to follow-up

(2) The subjects who asked to withdraw.

(3) The development of other diseases that may affect the outcome of the patient's treatment.

\subsubsection{Sample size calculation}

The sample size was calculated using Stata with a sampling error of 0.05 , a power of $80 \%$, and a nonresponse rate of $10.0 \%$ and assuming $15 \%$ score difference before and after the intervention. At least 250 patients were calculated as the sample size, and the same number was calculated for the control group. Based on the above assumptions, the minimum sample size for this study was calculated to be 500 patients.

\subsubsection{Research design}

According to the inclusion and exclusion criteria, the research participants were strictly screened. The present study was a randomized, controlled, parallel-group comparison trial with evaluators blinded to the allocation. The participants were randomly assigned to the routine health education group or the health management platform group and stratified by gender. To reduce potential contamination, any couples 
enrolled in the present study were randomized into the same group. The course was managed by an independent staff member not involved in the study. The patients in the routine health education group received health education management from clinicians according to the American Association for the Study of Liver Diseases (AASLD) the Practice Guidance for the Prevention and Treatment of Nonalcoholic Fatty Liver Disease (2017 edition)[27]. In addition to routine management, the health management platform group also received guidance and support from the health management platform, and the management norms were formulated by the platform expert group. The health management platform was developed on the basis of electronic health records by Beijing Medical Star Company. The Software as a Service (SaaS) mode was mainly adopted. Patient could use mobile phones to login to the patient-side app. Health management platform group members needed to first install the health management app on their mobile phones and receive relevant training. The platform model adopted unified membership management by establishing a digitized database to collect the information of the participants, formulate the individual therapeutic schedule for each participant and monitor their conditions. The subjects of the health management platform group were treated for NAFLD for 24 weeks. To encourage participants to complete the platform health management protocols, we offer a $10 \%$ discount on medical examination fees. All subjects were required to complete an examination of relevant observation and evaluation indicators and fill in the scale the 24th week after baseline regarding chronic disease management.

The technical roadmap is shown in Figure 1.

In this study, the service flow was divided into three steps: group archiving evaluation, intervention phase planning, and evaluation, providing closed-loop services for the prevention and rehabilitation of NAFLD. After introducing the new management model and service, patients were registered on the health management platform, and the management service package to be introduced was initially selected. The health management content accepted by the members of the platform management team mainly included the following parts:

(1) Establishment of electronic health records.

The collected baseline health information and related examination results were recorded in the health management files to provide the basis for health assessment and health intervention. The related management team includes chief experts, attending doctors, medical consultants, health consultants and so on.

(2) Individualized health assessment management.

According to the data of relevant indicators in health records, the health status of the subjects was evaluated, the main problems of the subjects were analyzed, the disease risks were determined, and the aspects to be improved and the matters needing attention were analyzed. Informing patients of the results of the examination and communicating and explaining them face to face can help the patients 
understand the significance of health indicators and encourage the subjects to actively manage their own health according to their health records.

(3) Health intervention management

$\otimes$ Dietary intervention management

According to the specific conditions of the study subjects, dietary guidance was provided to encourage the study subjects to change their bad eating habits; eat more vegetables, fruits and grains regularly; strictly control the intake of animal fat and cholesterol; limit sweets; control salt intake; avoid fried food and high-calorie foods; quit smoking and consuming alcohol; and avoid overeating.

\Sports Intervention Management

According to the physical activity level and physical condition of the subjects, the types, frequency and time of exercise were suggested. Adjustment suggestions were made in stages. The subjects were asked to perform aerobic exercises, such as jogging, walking, swimming and so on, at least four times a week for at least 40 minutes each time. The participants were shown various online videos, pictures and text so the subjects could learn and practice by themselves.

\section{$\otimes$ Cognitive intervention management}

Relevant NAFLD text-, film- and television-based guidance and other materials to publicize NAFLD-related knowledge and self-management-related knowledge were provided to participants. The content included the knowledge of NAFLD's etiology, prognosis, treatment principles and preventive measures and enabled the subjects to quickly acquire relevant knowledge using their free time.

\section{\Care}

Once a day, the health platform automatically sends out a form inquiring whether the participant was experiencing any discomfort symptoms. If the patient did not answer, the round was completed. If the answer was yes, the platform automatically sent out the inquiry form for the patient to complete. The content of the inquiry form was determined by the patient's current health status or illness, and the management team checked and completed the inquiry form for timely processing. In addition, weather change care, recent epidemic reminders, exercise, diet, exercise, psychological care, sleep care and so on were systematically promoted.

\section{\Appointment}

When the patient suffered from diseases, acute episodes of chronic diseases, complications, etc., general practitioners and hepatologists who treat patients with NAFLD determined whether hospitalization was necessary. The doctor confirmed the patients' applications for appointments regularly according to his or her working schedule. 
(4) The patient carried out the plan and uploaded it to receive feedback guidance.

(5) The team of doctors regularly evaluated and adjusted the plan according to the patient's health data.

(6) Follow-up.

Through the management platform for real-time online consultation (words, pictures, voice, etc.), patients carry out online follow-up; understand their diet, exercise, and psychological situation; facilitate timely detection of problems; and provide reminders and corrections. Moreover, according to the frequency of follow-up determined by the health plan, the system automatically sends reminders to patients. The health consultant checks the unfinished follow-up scale at any time and urges patients to complete the scale. This method can establish a closer relationship between the research object and the researchers and reduce the dropout rate.

\subsection{Basic demographic information survey}

The basic demographic information collected through questionnaires included name, sex, birth date, education level, marital status, employment, lifestyle factors (smoking, alcohol consumption), medication use and disease history.

2.4 Observation and evaluation index

\subsubsection{Safety indicators}

(1) The blood tests included hemoglobin $(\mathrm{Hb})$, red blood cell count (RBC), leukocyte count (WBC), platelet count (PLT), lymphocyte proportion (Lym), neutrophil ratio (Neu) and other items.

(2) The renal function test included serum creatinine (Scr) blood urea nitrogen (BUN), blood uric acid (BUA) and other indices.

\subsubsection{Basic indicators}

(1) Weight

The subjects removed their coats and shoes, stood naturally on an automatic body mass index (BMI) measuring stadiometer (BSM370, Korea), and kept their body stable. Once the reading became stable, the weight was read $(\mathrm{kg})$; the data were accurate to $0.1 \mathrm{~kg}$.

(2) Waist circumference (WC)

An anthropometric tape was used to measure waist circumference (WC). WC was measured midway between the lowest rib margin and the iliac crest. During the measurement, the subjects stood upright, relaxed their abdominal, relaxed their arms, stood with their feet together, breathed gently, and did not hold their breath; the data were accurate to $0.1 \mathrm{~cm}$. 
(3) Hip circumference (HIPS)

An anthropometric tape was used to measure hip circumference. Hip circumference was measured at the maximum protuberance of the buttocks in a standing position, and the data were accurate to $0.1 \mathrm{~cm}$.

(4) Body mass index (BMI)

Standing height without shoes was measured to the nearest $0.1 \mathrm{~cm}$ using an automatic BMI measuring stadiometer (BSM370, Korea) with a precision of $0.1 \mathrm{~cm}$. BMI was calculated as weight $(\mathrm{kg}) /$ [height $(\mathrm{m})]^{2}$, and the data were accurate to 0.01 . BMI was categorized as follows: slim, $\mathrm{BMI}<18.5$; normal weight, $18.5 \leq \mathrm{BMI}<22.9$; overweight, $23 \leq \mathrm{BMI} \leq 27.9$; and obesity, $\mathrm{BMI} \geq 28$.

(5) Blood pressure (BP)

Blood pressure, including systolic blood pressure (SBP) and diastolic blood pressure (DBP), was measured twice in a comfortable quiet sitting position by a calibrated mercury Omron electronic sphygmomanometer (model: HBP-1300) with the right arm supported at the level of the heart after at least 5 minutes' rest.

All equipment used in the study was calibrated. All measurements were performed by a single individual to reduce the system error.

\subsubsection{Laboratory parameters}

The examinees fasted for at least 8 hours; blood was taken from the cubital vein in the morning and was tested immediately after centrifugation with a Hitachi 7600 automatic biochemical analyzer.

(1) Liver function tests included alanine aminotransferase (ALT), aspartate aminotransferase (AST), and AST/ALT values.

(2) Four lipid indicators including triglycerides (TGs), total cholesterol (CHOL), high-density lipoprotein (HDL-C), low-density lipoprotein (LDL-C) were assessed in addition to other parameters.

(3) Fasting plasma glucose (FPG)

All of the above clinical parameters were assessed at the Department of Laboratory Medicine, Qilu Hospital, Shandong University, China.

\subsubsection{Body composition}

The analysis of body composition was performed using a body composition analyzer (InBody) with multifrequency. Bioelectrical impedance measurement technology can provide detailed analysis reports of human body water, fat and protein components and is the most effective new indicator for analyzing 
and managing the nutrition and water status of patients. The measured indices included body fat content $(\mathrm{kg})$, skeletal muscle content $(\mathrm{kg})$, and visceral fat area $\left(\mathrm{cm}^{2}\right)$.

\subsubsection{Controlled attenuation parameter (CAP)}

The CAP is a system that measures the degree of ultrasound attenuation by hepatic fat using a process based on vibration control transient elastography [28-29]. CAP was measured by one experienced technician using the FibroScan-502 ultrasound diagnostic instrument (Echosens, Paris, France). During the test, the subjects lie on their backs and hold their heads in their right hands. The measurement points are usually between the axillary midline of the right 7-9 rib space and the axillary front line. Keeping the M probe perpendicular to the skin surface and avoiding the structure of large blood vessels, each subject was successfully measured more than 10 times. After the measurement, the instrument automatically took the median of the measurement as the final measurement result. An effective measurement was considered when the ratio of interquartile range to the median of all measurements (IQR/med) was less than $30 \%$. The success rate (number of successful tests/total tests) should be more than $60 \%$.

\subsubsection{Quality of life assessment}

The Quality of Life and Health Survey Scale (SF-36) is also called the Medical Outcomes Study Shortform 36 (MOSSF-36). General health status parameters designed for population surveys or evaluative studies of health policies are also used to measure outcomes associated with certain diseases in clinical practice and research. The scale includes 36 items, including physiological function (PF), role physical $(\mathrm{RP})$, role emotional (RE), vitality (VT), mental health $(\mathrm{MH})$, social functioning (SF), body pain (BP), general health status $(\mathrm{GH})$ and 8 other categories involving physical and mental health. Each domain was scored on a standardized scale with values ranging between 0 and 100, where 0 represents the lowest and 100 represents the highest quality of life. Sf-36 is one of the most commonly used international standardized measurement tools to assess quality of life. The Chinese version of SF-36 has been translated and applied for many years, and its reliability and validity have been verified. In a previous study, the Cronbach's alpha reliability coefficients ranged between 0.72 and 0.88 , and the test-retest correlation coefficients ranged between 0.66 and 0.94 [30]. Before using the Chinese version of the SF-36 scale, 32 participants were randomly selected from the included subjects for the preliminary experiment to test the reliability and validity of the SF-36 scale in NAFLD patients. The preliminary results showed that Cronbach's alpha $=0.720, \mathrm{KMO}=0.647$, Bartlett sphericity test $=0.000$, and three common factors produced by factor analysis could explain $67.67 \%$ of the total variation. The overall reliability and validity of the scale were tested. The scale can be used to investigate the quality of life and health status of patients with NAFLD. In this study, eight dimensions of the SF-36 scale were included in the score; the higher the score, the better the quality of life in the corresponding dimensions.

\subsection{Quality Control}

Participants needed to undergo unified training; after the training, the researchers were assessed, and the consistency of the researchers was tested by the kappa test. Only when kappa $>0.6$ could the research be 
carried out. The participants' unified criteria for judgment confirm the consistency of the selection of research subjects; the intervention and program; and the consistency, reliability and integrity of the outcome evaluation indicators. The researchers were responsible for monitoring and reducing the rate of missing visits. The research data and summary materials are being managed by the health management center of Qilu Hospital, Shandong University. The center is subordinate to the third class hospital of the government, and authenticity and reliability were ensured.

\subsection{Statistical analysis}

Data were collected and a database was established. SPSS 22.0 software was used to analyze the data. The counting data are expressed as the constituent ratio and rate (\%). Metrological data are expressed as the mean \pm standard deviation $(X \pm S)$, and categorized variables are expressed as numbers. According to the specific data type, the corresponding analysis methods were adopted. Measurement data that satisfied both normal distribution and homogeneity of variance were analyzed by a t-test or analysis of variance. For non-normally distributed data, the natural logarithms were used for further analysis, if the data did not satisfy the requirements, a rank sum test was used. The counting data were tested by the $\chi^{2}-$ test or Fisher exact test, and the comparison between groups was performed by one-way ANOVA or Nemenyi test.

\section{Results}

After screening according to the inclusion and exclusion criteria, 600 eligible patients with nonalcoholic fatty liver disease were recruited. A total of 519 patients completed the follow-up, including 261 in the health management platform group and 258 in the routine health education group. During the follow-up period, 81 people were lost to follow-up for various reasons. The rate of lost visits was $13.5 \%$. Among the participants who were lost to follow-up, 39 were in the health management platform group and 42 were in the routine health education group. The main reasons for missing the visit were withdrawal and hospitalization. After statistical analysis of the missing population and those who completed the followup, there was no significant difference in sociodemographic information, indicating that the missing population would not bias the results. There was no significant difference in age distribution, sex composition or NAFLD level between the routine health education group and the health management platform group $(P>0.05)$. The basic information included in the study is shown in Table 1.

\subsection{Test results of basic physical indicators before and after the intervention}

At the end of the study, there was no significant change in the results of the basic physical indicators in the routine health education group before and after the test $(P>0.05)$.

The body weight and BMI of the subjects in the health management platform group after the intervention were significantly lower than those before the intervention ( $P=0.004$ and $P=0.001$, respectively). 
Intergroup analysis showed that the weight and BMI of the subjects in the health management platform group were significantly lower than those in the routine health education group $(P=0.047$ and $P=0.031$, respectively), as shown in Tables 2 and 5.

3.2 Comparison and analysis of blood biochemical indicators and indicators of body composition before and after the intervention

The statistical analysis of blood biochemical indicators and indicators of body composition revealed that at the end of the study, in the health management platform group, $\mathrm{CHOL}$ and HDL-c were significantly higher than those before the intervention ( $P=0.022$ and $P=0.031$, respectively), while body fat content, visceral fat area, CAP and AST/ALT were significantly lower $(P=0.001,0.003,0.049$ and $P=0.038$, respectively). In the routine health education group, there were no significant changes in the results of liver function, FPG, CAP or AST / ALT $(P>0.05)$, but $\mathrm{CHOL}$ was significantly higher than the level before the intervention $(P<=0.004)$.

Intergroup analysis showed that the health management platform group had higher HDL-c levels and lower visceral fat area, AST/ALT values and CAP than the routine health education group $(P=0.046$, $0.047,0.037$ and $P=0.044$, respectively). The details are shown in Tables 3 and 5 .

\subsection{SF-36 scale score results}

Statistical analysis of the scores of the SF-36 scale before and after the intervention showed that there was no significant change in the scores in any dimensions in the subjects in the routine health education group ( $P>0.05)$, while the scores of the RP, VT and GH dimensions of the subjects in the health management platform group increased significantly $(P=0.027,0.001$ and 0.044 , respectively). The VT dimension scores of the subjects in the health management platform group were significantly higher than those in the routine health education group $(P=0.001)$. The details are shown in Tables 4 and 5 .

\section{Discussion}

In this study, body weight, BMI, WC, HIPS and waist-hip ratio were used as some of the evaluation indicators of the NAFLD intervention effect. Additionally, a human body component analyzer and multifrequency bioelectrical impedance measurement technology were used to accurately quantify body fat content, skeletal muscle content and visceral fat area. After 24 weeks of the platform management intervention, the results showed that the weight, BMI, fat content and visceral fat area of the NAFLD patients in the health management platform group were significantly lower than those before intervention, while there was no significant reduction in these parameters in the conventional health management group. The body weight and BMI of the subjects in the health management platform group were significantly lower than those in the routine health education group, which indicated that health management platform could effectively reduce body fat accumulation and play a positive role in the prevention and treatment of NAFLD. 
In recent years, AST/ALT has received increasing attention in the context of the diagnosis and prognosis of liver diseases. The ratio of AST/ALT directly reflects the damage of hepatocytes. The results of this study showed that the levels of $\mathrm{CHOL}$ and $\mathrm{HDL}-\mathrm{c}$ after the intervention in the health management platform group were significantly higher than those before intervention; TGs and LDL-c showed no significant changes, but AST/ALT was significantly lower than that before the intervention. The HDL-C value in the health management platform group was significantly higher than that in the routine health management group. Combined with the decrease in visceral fat area in the body composition analysis, it is speculated that the increase in HDL-c is beneficial for the transfer of fat from the liver and the decrease in hepatocyte damage, which may be manifested as a decrease in AST/ALT when the total amount of TGs remains unchanged. Although $\mathrm{CHOL}$ also increased, it is speculated that the reason may be due to the increase in HDL-c content.

CAP, as a simple and noninvasive new technique for the quantitative assessment of liver fat content, is reported to be associated with the grade of steatosis as assessed by ultrasound as well as histological steatosis severity and is significantly and positively correlated with BMI [31]. In our study, the median CAP of the platform management group decreased significantly by $16.3 \%$ from $294.09 \pm 19.91 \mathrm{~dB} / \mathrm{m}(216-$ $400 \mathrm{~dB} / \mathrm{m})$ to $246.40 \pm 17.74 \mathrm{~dB} / \mathrm{m}(100-353 \mathrm{~dB} / \mathrm{m})$, while there was no significant change in the routine health education group. This finding reflects the substantial effect of the health management platform from one perspective.

The SF-36 scale can be used to evaluate the quality of life and health status of the human body based on physiological, psychological and social functions. The scale can be used in people with different diseases [32-33] and has been widely recognized and applied in Europe and the United States. Among the eight dimensions of the SF-36 scale, PF, RP, BP and GH are defined as physiological factors, while RE, VT, $\mathrm{MH}$ and SF are defined as psychological factors [34-35]. This study evaluated the impact of a health education management model on patients with NAFLD. The results showed that the scores of the RP, VT and $\mathrm{GH}$ dimensions in the platform management group after the intervention were significantly higher than those before the intervention, while the scores of the eight dimensions of quality of life in the routine health education group were not significantly changed. In the intergroup analysis, the VT dimension scores of the health management platform group were significantly higher than those in the routine health education group, indicating the effective implementation of chronic disease management in this study. An earlier study by Mehmet Sayiner and AA Dan [36,37], which reported a similar result, demonstrated that disease management plays an active role in improving the physiological health and $\mathrm{MH}$ of the NAFLD population.

The results showed that the "Internet plus medical"-based health management service model of NAFLD could help control body weight $(-3.80 \pm 3.11 \mathrm{vs}-0.12 \pm 3.42, P=0.047)$, body fat content $(-2.24 \pm 2.58$ vs $-0.86 \pm 2.78, P=0.194)$, and $\mathrm{BMI}(-1.73 \pm 1.20$ vs $-0.14 \pm 1.68, P=0.031)$ and improve liver function and quality of life in patients with NAFLD in terms of physiological role $(96.59 \pm 11.31$ vs $90.69 \pm 13.49$, $P=0.027)$, vitality $(86.57 \pm 6.07$ vs $81.94 \pm 5.78, P=0.001)$ and general health $(78.05 \pm 6.35 \mathrm{vs} 73.61 \pm 10.12$, $P=0.044$ ) to achieve the effect of preventing and treating NAFLD. The findings can provide a reference for 
exploring the management model of NAFLD and promoting the health status of patients. In conclusion, the model is feasible and effective in the prevention and treatment of NAFLD by reducing the level of major risk factors in patients with NAFLD and improving their health and quality of life.

The "Internet plus medical"-based health management service model utilizes a "multidisciplinary team approach", which makes medical resources break through the time and space constraints of the traditional health service mode and seamlessly connect with existing medical institutions, providing continuous health services. For patients with NAFLD, the health management mobile phone app platform increases their access to health management knowledge, enhances patients' awareness of selfmanagement, promotes information sharing between patients and medical teams, simplifies the medical procedures of patients with nonalcoholic fatty liver disease, and saves patients time and money. For medical institutions, the introduction of Internet technology into the management model of NAFLD can reduce the workload of nonalcoholic fatty liver disease management, help scientifically and rationally allocate medical resources, reduce the use of medical resources, ensure the continuity of the management institutions, and open a convenient door for two-way referral and graded diagnosis and treatment, which has greater social and economic benefits.

This study has some strengths and limitations. First, the participants in the study were recruited from health management centers or weight loss clubs. Therefore, there is inevitably a selection bias in this study. Second, there was a relatively small sample size, short management time and poor efficacy in observing blood lipids and other indicators, which led to differences between groups that were large but had $p$ values that were almost not significant. Finally, due to the practical difficulties in obtaining tissues from living individuals (liver biopsy is not widely accepted in China), liver biopsy is unsuitable for population screening. Ultrasound is less accurate than the gold standard of biopsy in the diagnosis of nonalcoholic fatty liver disease. In this study, the overall rate of missing interviews was $35.3 \%$ and that of the platform management group was $37.7 \%$. Half-way withdrawal of patients in the platform management group may be related to the use of the health management platform. In the later period, a telephone follow-up survey of the lost patients revealed that the main reason for the decrease in the frequency of patients using the health management platform and the final withdrawal was that it took more time. The mobile network health management system must be easy to use, provide help and guidance for patients to prevent them from discontinuing its use because of its complexity. The problem of missing data is common in observational studies and may lead to biased results. Subjects who did not complete an interview were excluded from the study, which also may have biased the results. In future studies, we will take measures to increase the response rate for interview completion. With respect to the process of using the platform, we will implement the following improvements. First, to make the system easier to use, we will further improve the system design, minimize the use barriers caused by technical reasons, and strengthen the management platform training so that patients will have a more comprehensive understanding of client functions. In addition, a more effective intervention mechanism and management process will be explored that allows an individualized intervention plan to be formulated for each patient and coordination among hospital staff teams to ensure ongoing care for participants. Additionally, the implementation of the intervention will be supervised to increase patients' 
compliance with the health management platform and reduce their use burden. Long-term research is needed to determine the effectiveness of this type of intervention platform over time not only on patient outcomes but also on the time and resources needed to maintain the intervention.

\section{Conclusions}

In conclusion, the "Internet plus medical"-based health management service model optimizes the management and operation process of patients with NAFLD, improves the feasibility, systematicness and intelligence of health management services, and provides active and full-course health management services for patients with NAFLD. The studied intervention is an evidence-based model of health education and management for Chinese NAFLD prevention that will be of great significance to improve the health of individuals in China.

\section{Declarations}

\section{Availability of data and materials}

The datasets used and/or analyzed during the current study are available from Health Management Center, QiLu Hospital of Shandong University, but restrictions apply on the availability of these data, which were used under license for the current study and thus are not publicly available. However, the data are available from the authors upon reasonable request and with the permission of Health Management Center, QiLu Hospital of Shandong University.

\section{Acknowledgments}

We greatly appreciate the time and effort of the participants of this study, especially the staff working at Health Management Center of QiLu Hospital of Shandong University.

\section{Funding}

There was no financial support.

\section{Author information}

\section{Affiliations}

Health Management Center, QiLu Hospital of Shandong University, Jinan, Shandong Province, China Yan Yang, Jun Dai, Wei Zhang, Shu-Qing Li, Xiang Min, Yan-Yan Wang, Juan Cao

School of Medicine, Shandong University Jinan 250012 China

Cui-Huan Tian 


\section{Corresponding author}

Correspondence to Yan Yang

\section{Author Contributions}

Yan Yang had full access to all of the data in the study and takes responsibility for the integrity of the data and the accuracy of the data analysis. Yan Yang participated in the study conception and design and revised the manuscript. Cui-Huan Tian, Xiang Min, Juan Cao and Jun Dai contributed to the conception of the study, collection and analysis of the data, and drafting of the manuscript. The analysis and interpretation of the data were performed by Wei Zhang, Shu-Qing Li and Yan-Yan Wang. All authors read and approved the final manuscript. The authors have also agreed to be accountable for all aspects of the work in regard to ensuring that questions related to the accuracy or integrity of any part of the work are appropriately investigated and resolved.

\section{Ethics declarations}

\section{Ethics declarationsand consent to participate}

Permission was obtained from the Ethics Committee of Qilu Hospital of Shandong University, China (NO: 2018021214). The date of approval by the ethics committee was 2018-02-12. All participants (18 years of age or older) agreed to participate voluntarily in the study and signed a consent form after receiving an explanation of the objectives and the process of the study. Data collection was anonymous, and confidentiality and privacy were respected.

\section{Consent for publication}

Not applicable.

\section{Competing interests}

The authors declare that they have no competing interests.

\section{Additional information}

\section{Publisher's Note}

Springer Nature remains neutral with regard to jurisdictional claims in published maps and institutional affiliations.

\section{Abbreviations}

NAFLD Non-alcoholic fatty liver disease

ALT Alanine transaminase 
AST Aspartate transaminase

BMI Body mass index

BP Blood pressure

FBG Fasting blood glucose

HDL-C High-density lipoprotein cholesterol

LAP Lipid accumulation product

TC Total cholesterol

TG Triglyceride

CHOL Total cholesterol

WC Waist circumference

HIPS Hip circumference

WHR Waist-hip ratio

SF-36 Short Form-36 Health Survey

CAP Controlled attenuation parameter

SaaS Software as a Service

APP Application

\section{References}

1. Younossi ZM, Koenig AB, Abdelatif D, Fazel Y, Henry L, Wymer M. Global epidemiology of nonalcoholic fatty liver disease-meta-analytic assessment of prevalence, incidence, and outcomes. Hepatology. 2016;64:73-84.

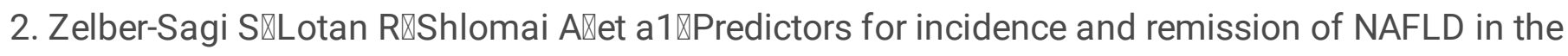
general population during a seven-year prospective follow - up[J] $₫ J$ Hepatol $₫ 2012 \varangle 56(5) \varangle 1145-1151 \rrbracket$

3. Fan JG. Epidemiology of alcoholic and nonalcoholic fatty liver disease in China. J Gastroenterol Hepatol. 2013;28(Suppl 1):11-7.

4. Li Z, Xue J, Chen P, Chen L, Yan S, Liu L. Prevalence of nonalcoholic fatty liver disease in mainland of China: a meta-analysis of published studies.J Gastroenterol Hepatol. 2014;29:42-51. 
5. Yu J, Shen J, Sun TT, Zhang X, Wong N. Obesity, insulin resistance, NASH and hepatocellular carcinoma. Semin Cancer Biol. 2013;23:483-491.

6. Wong RJ, Aguilar M, Cheung R, Perumpail RB, Harrison SA, Younossi ZM, Ahmed A. Nonalcoholic steatohepatitis is the second leading etiology of liver disease among adults awaiting liver transplantation in the United States. Gastroenterology. 2015;148:547-555.

7. Fukuda T, Hamaguchi M, Kojima T, Hashimoto Y, Ohbora A, Kato T, Nakamura N, Fukui M. The impact of non-alcoholic fatty liver disease on incident type 2 diabetes mellitus in non-overweight individuals. Liver Int.2016;36:275-283.

8. Ballestri S, Zona S, Targher G, Romagnoli D, Baldelli E, Nascimbeni F, Roverato A, Guaraldi G, Lonardo A. Nonalcoholic fatty liver disease is associated with an almost twofold increased risk of incident type 2 diabetes and metabolic syndrome. Evidence from a systematic review and meta-analysis. $J$ Gastroenterol Hepatol. 2016;31:936-944.

9. Musso G, Gambino R, Tabibian JH, Ekstedt M, Kechagias S, Hamaguchi M, Hultcrantz R, Hagstrom H, Yoon SK, Charatcharoenwitthaya $\mathrm{P}$, et al. Association of non-alcoholic fatty liver disease with chronic kidney disease: a systematic review and meta-analysis. PLoS Med. 2014;11:e1001680.

10. Targher G, Byrne CD, Lonardo A, Zoppini G, Barbui C. Non-alcoholic fatty liver disease and risk of incident cardiovascular disease: a meta-analysis. J Hepatol. 2016;65:589-600.

11. Targher G, Bertolini L, Padovani R, et al. Prevalence of nonalcoholic fatty liver disease and its association with cardiovascular disease among type 2 diabetic patients. Diabetes Care. 2007;30(5):1212-1218.

12. Targher G, Bertolini L, Rodella S, et al. Non-alcoholic fatty liver disease is independently associated with an increased prevalence of chronic kidney disease and proliferative/laser-treated retinopathy in type 2 diabetic patients. Diabetologia. 2008;51(3):444-450.

13. Adams LA, Harmsen S, St Sauver JL, et al. Nonalcoholic fatty liver disease increases risk of death among patients with diabetes: a community-based cohort study. Am J Gastroenterol. 2010;105(7):1567-1573.

14. Mlynarski L, Schlesinger $D$, Lotan R, et al. Non-alcoholic fatty liver disease is not associated with a lower health perception. World J Gastroenterol. 2016;22(17):4362-4372.

15. Centis $E$, Moscatiello S, Bugianesi $E$, et al. Stage of change and motivation to healthier lifestyle in non-alcoholic fatty liver disease. J Hepatol. 2013;58(4):771-777.

16. Zelber-Sagi S, Bord S, Dror-Lavi G, et al. Role of illness perception and self-efficacy in lifestyle modification among non-alcoholic fatty liver disease patients. World $\mathrm{J}$ Gastroenterol. 2017;23(10):1881-1890.

17. Bellentani S, Scaglioni F, Marino M, Bedogni G. Epidemiology of nonalcoholic fatty liver disease. Dig Dis 2010;28:155-61

18. Clark JM. The epidemiology of nonalcoholic fatty liver disease in adults. J Clin Gastroenterol 2006;40 Suppl 1:S5-10. 
19. LaBrecque DR, Abbas Z, Anania F, Ferenci P, Khan AG, Goh K-L, Hamid SS, Isakov V, Lizarzabal M, Chalasani N, Younossi Z, Lavine JE, Diehl AM, Brunt EM, Cusi K, Charlton M, Sanyal AJ. The diagnosis and management of non-alcoholic fatty liver disease: Practice Guideline by the American Association for the Study of Liver Diseases, American College of Gastroenterology, and the American Gastroenterological Association. Hepatology 2012;55:2005-23.

20. Peñaranda MM. World Gastroenterology Organisation global guidelines: Nonalcoholic fatty liver disease and nonalcoholic steatohepatitis. J Clin Gastroenterol 2014;48:467-73.

21. Zhang W, Ohira T, Yasumura S, et al. Effects of socioeconomic factors on cardiovascular-related symptoms among residents in Fukushima after the Great East Japan Earthquake: a cross-sectional study using data from the Fukushima Health Management Survey.BMJ Open. 2017;7(6):e014077.

22. Zheng L, Wang Y, Hao S, et al. Web-based Real-Time Case Finding for the Population Health Management of Patients With Diabetes Mellitus: A Prospective Validation of the Natural Language Processing-Based Algorithm With Statewide Electronic Medical Records. JMIR Med Inform. 2016;4(4):e37.

23. Peyman, N., Rezai-Rad, M., Tehrani, H. et al. Digital Media-based Health Intervention on the promotion of Women's physical activity: a quasi-experimental study. BMC Public Health 18, 134 (2018). https://doi.org/10.1186/s12889-018-5025-5.

24. Sarkisova V. Quality of nursing care, availability and accessibility of treatment and prevention of communicable and chronic diseases. Int J Nurs Sci 2017;4:.

25. World Health Organisationm Health-New Horizons for Health Through Mobile Technologies. Geneva: WHO; 2011

26. Zhijie W, Qing G. Study on the Development Strategy of Health Management Industry in big data era. Res Health Eco 2014;14-6.

27. Chalasani N, Younossi Z, Lavine J E, et al. The Diagnosis and Management of Nonalcoholic Fatty Liver Disease: Practice Guidance from the American Association for the Study of Liver Diseases[J]. Hepatology, 2017.

28. Kumar M, Rastogi A, Singh T, Behari C, Gupta E, Garg H, et al. Controlled attenuation parameter for non-invasive assessment of hepatic steatosis: does etiology affect performance. J Gastroenterol Hepatol. 2013;28:1194-1201.

29. Castera L, Forns X, Alberti A. Non-invasive evaluation of liver fibrosis using transient elastography. J Hepatol. 2008;48:835-847.

30. Li L, Wang H and Shen Y: Development and psychometric tests of a Chinese version of the SF-36 health survey scales. Zhonghua Yu Fang Yi Xue Za Zhi 36: 109-113, 2002.

31. Ahn J M , Paik Y H, Min S Y, et al. Relationship between Controlled Attenuation Parameter and Hepatic Steatosis as Assessed by Ultrasound in Alcoholic or Nonalcoholic Fatty Liver Disease.[J]. Gut \& Liver, 2016, 10(2):295-302.

32. Wafa hamdi, Azzouz D, Ghannouchi MM, et al. Health-related quality of life assessment on 100 tunisian patients with ankylosing spondylitis using the SF-36 survey[J]. Oman Med J,2012, 
27(6):455-460.

33. Jajić Z, Rajnpreht, I, Kovačić $\mathrm{N} \rrbracket$ et al. Which clinical variables have the most significant correlation with quality of life evaluated by SF-36 survey in Croatian cohort of patient with ankylosing spondylitis and psoriatic arthritis[J] Rheumatol Int,2012,32(11):3471-3479.

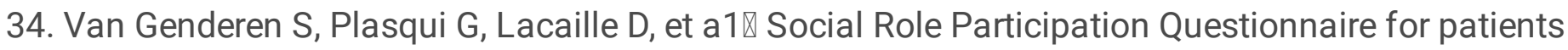
with ankylosing spondylitis: translation into Dutch, reliability and construct validity[J]. RMD Open, 2016,2(1):e000177.

35. Mörck B, Pullerits R, Geijer M,et al. Infliximab dose reduction sustains the clinical treatment effect in active HLAB27 positive ankylosing spondylitis: a two-year pilot study[J]. Mediators Inflamm, 2013:289845.

36. Sayiner M, Stepanova M , Pham H , et al. Assessment of health utilities and quality of life in patients with non-alcoholic fatty liver disease[J]. Bmj Open Gastroenterology, 2016, 3(1):e000106.

37. Dan AA, Kallman JB, Wheeler A, et al. Health-related quality of life in patients with non-alcoholic fatty liver disease. Aliment Pharmacol Ther 2007;26:815-20.

\section{Tables}

Table 1. Basic information of participants 


\begin{tabular}{lllr}
\hline Characteristics & Routine health education & Health management & $P$ \\
group & platform & group & value \\
& &
\end{tabular}

Number

Sex

Men (n \%)

Women (n \%)

Age (years)

NAFLD degree

Mild (n \%)

Moderate (n \%)

Severe (n \%)

Marital status

Unmarried (n \%)

Married (n \%)

Widowed (n \%)

Divorced (n \%)

Ethnicity

Han Chinese (n

\%)

Minority Chinese (n \%)

Education

Elementary school (n \%)

Junior high school (n \%)

Senior high school (n \%)

College or above (n \%)

Nature of work
258

261

0.330

161(62.40)

136(52.11)

97(37.60)

125(47.89)

$44.71 \pm 10.81$

$43.15 \pm 11.52$

0.254

0.376

155(60.01)

157(60.15)

73(28.29)

87(33.33)

$30(11.70)$

17(6.52)

0.047

14(5.43)

47(18.01)

237(91.86)

204(78.16)

$2(0.77)$

2(0.76)

5(1.94)

8(3.07)

0.394

$250(96.89)$

256(98.08)

8(3.11)

5(1.92)

3(1.16)

$0(0)$

$22(8.52)$

29(11.11)

60(23.26)

$50(19.16)$

173(67.08)

$182(69.73)$
0.069

0.101 
Physical labor (n \%)

Nonmanual labor (n \%)

Smoking

Yes (n \%)

No (n \%)
$10(3.88)$

248(96.12)

$20(7.75)$

238(92.25)
28(10.73)

$233(89.27)$

0.788

23(8.81)

238(91.19)

Categorical values are presented as relative frequencies.

Notes: The results of the $\chi^{2}$-test showed that $P=0.330>0.05$. There was no significant difference in sex composition between the routine health education group and the health management platform group.

The Kruskal-Wallis $\mathrm{H}$ rank sum test showed that $P=0.254>0.05$, and there was no significant difference in the age of the subjects between the routine health education group and the health management platform group. Fisher's exact test showed that $P=0.376>0.05$; there was no significant difference in the degree of NAFLD between the routine health education group and the health management platform group.

Table 2. Comparison of the results of basic indicators before and after the intervention 


\begin{tabular}{|c|c|c|c|c|c|c|}
\hline \multirow{5}{*}{ Variable } & \multicolumn{2}{|c|}{ Routine health education group } & \multirow{5}{*}{$P$ value } & Health manage & ement platform & \multirow{5}{*}{$P$ value } \\
\hline & & & & group & & \\
\hline & \multirow{2}{*}{ Before } & \multirow{2}{*}{ After } & & \multicolumn{2}{|c|}{ After } & \\
\hline & & & & \multirow[t]{2}{*}{ intervention } & \multirow{2}{*}{ intervention } & \\
\hline & \multicolumn{2}{|c|}{ intervention intervention } & & & & \\
\hline Weight $(\mathrm{kg})$ & $80.19 \pm 10.63$ & $80.07 \pm 11.38$ & 0.354 & $79.33 \pm 9.84$ & $75.23 \pm 11.62$ & $0.004^{*}$ \\
\hline WC (cm) & $97.04 \pm 7.62$ & $96.52 \pm 7.47$ & $0.412-$ & $97.65 \pm 8.15$ & $96.38 \pm 7.83$ & 0.071 \\
\hline HIPS (cm) & $104.63 \pm 5.92$ & $103.72 \pm 7.29$ & 0.786 & $103.72 \pm 7.29$ & $102.58 \pm 6.02$ & 0.783 \\
\hline WHR & $0.95 \pm 0.07$ & $0.95 \pm 0.05$ & 0.596 & $0.96 \pm 0.05$ & $0.94 \pm 0.04$ & 0.414 \\
\hline BMI & $28.58 \pm 3.54$ & $28.44 \pm 3.41$ & 0.121 & $28.76 \pm 2.96$ & $27.03 \pm 3.11$ & $0.001^{*}$ \\
\hline SBP & $124.78 \pm 18.91$ & $126.23 \pm 11.32$ & 0.890 & $121.14 \pm 10.74$ & $123.22 \pm 15.17$ & 0.126 \\
\hline DBP & $72.91 \pm 13.12$ & $76.53 \pm 11.41$ & 0.411 & $80.80 \pm 10.41$ & $83.11 \pm 10.37$ & 0.548 \\
\hline
\end{tabular}

T-test with paired samples. $P<0.05$, with a significant difference.

Data are expressed as the means (standard deviations) for continuous variables or frequencies (percentages, \%) for categorical variables.

*Statistics by t-test for continuous variables and chi-square test for categorical variables.

WC, Waist Circumference; HIPS, Hip Circumference; WHR, Waist Hip Ratio; BMI, Body Mass Index; SBP, systolic blood pressure; DBP diastolic blood pressure;

Table 3. Comparison of the results of blood biochemical indicators and indicators of body composition before and after the intervention 


\begin{tabular}{|c|c|c|c|c|}
\hline \multirow{5}{*}{ Variable } & \multirow{3}{*}{$\begin{array}{l}\text { Routine health education } \\
\text { group }\end{array}$} & \multirow{5}{*}{$\begin{array}{l}P \\
\text { value }\end{array}$} & Health management platform & \multirow{5}{*}{$P$ value } \\
\hline & & & group & \\
\hline & & & Before After & \\
\hline & Before After & & \multirow[t]{2}{*}{ intervention intervention } & \\
\hline & Intervention intervention & & & \\
\hline ALT (U/L) & $51.16 \pm 47.68 \quad 42.53 \pm 28.77$ & 0.574 & $54.04 \pm 57.23 \quad 40.15 \pm 29.78$ & 0.068 \\
\hline AST(U/L) & $35.81 \pm 35.15 \quad 30.19 \pm 14.67$ & $0.486-$ & $35.79 \pm 30.18 \quad 29.44 \pm 15.13$ & 0.577 \\
\hline AST/ALT & $0.84 \pm 0.29 \quad 0.80 \pm 0.35$ & 0.284 & $0.98 \pm 0.27 \quad 0.80 \pm 0.33$ & $0.038^{*}$ \\
\hline GGT & $42.36 \pm 15.12 \quad 41.85 \pm 17.91$ & 0.599 & $44.15 \pm 17.83 \quad 43.07 \pm 21.32$ & 0.411 \\
\hline FPG & $5.27 \pm 1.055 .71 \pm 1.62$ & 0.171 & $5.08 \pm 0.715 .57 \pm 1.69$ & 0.053 \\
\hline TG & $2.31 \pm 1.79 \quad 2.12 \pm 1.54$ & 0.218 & $2.42 \pm 1.85 \quad 2.17 \pm 1.37$ & 0.616 \\
\hline CHOL & $4.88 \pm 1.195 .26 \pm 1.09$ & $0.004^{*}$ & $5.16 \pm 1.20 \quad 5.33 \pm 1.18$ & $0.022^{*}$ \\
\hline HDL-c & $1.37 \pm 0.53 \quad 1.29 \pm 0.29$ & 0.775 & $1.05 \pm 0.39 \quad 1.25 \pm 0.28$ & $0.031^{*}$ \\
\hline $\mathrm{CAP}(\mathrm{dB} / \mathrm{m})$ & $292.13 \pm 16.70 \quad 288.24 \pm 19.11$ & 0.564 & $294.09 \pm 19.91$ & $0.049^{*}$ \\
\hline LDL-C & $3.32 \pm 0.75 \quad 3.39 \pm 0.59$ & 0.512 & $246.40 \pm 17.74$ & 0.246 \\
\hline Body fat content (kg) & $29.55 \pm 6.38 \quad 28.19 \pm 6.23$ & 0.157 & $3.25 \pm 0.78 \quad 3.28 \pm 0.79$ & $0.001^{*}$ \\
\hline Skeletal muscle content & $27.59 \pm 5.27 \quad 28.15 \pm 5.95$ & 0.081 & $28.95 \pm 6.01 \quad 26.91 \pm 5.73$ & 0.726 \\
\hline$(\mathrm{kg})$ & $136.02 \pm 48.19 \quad 135.48 \pm 37.47$ & 0.613 & $26.15 \pm 5.63 \quad 26.38 \pm 5.61$ & $0.003^{*}$ \\
\hline \multirow[t]{2}{*}{ Visceral fat area $\left(\mathrm{cm}^{2}\right)$} & & & \multicolumn{2}{|l|}{$142.12 \pm 31.97$} \\
\hline & & & \multicolumn{2}{|l|}{$130.15 \pm 32.91$} \\
\hline
\end{tabular}

ALT, Alanine transaminase; GGT, Glutamyl transpeptidase; AST, Aspartate transaminase; FBG, Fasting blood glucose; HDL-C, High-density lipoprotein cholesterol; LDL-c, Low-density lipoprotein cholesterol; CAP, Controlled attenuation parameter; TG, Triglyceride; CHOL, Cholesterol Total.

Table 4. Comparison of the SF-36 scores between the routine health education group and the health management platform group before and after the intervention (mean \pm s). 


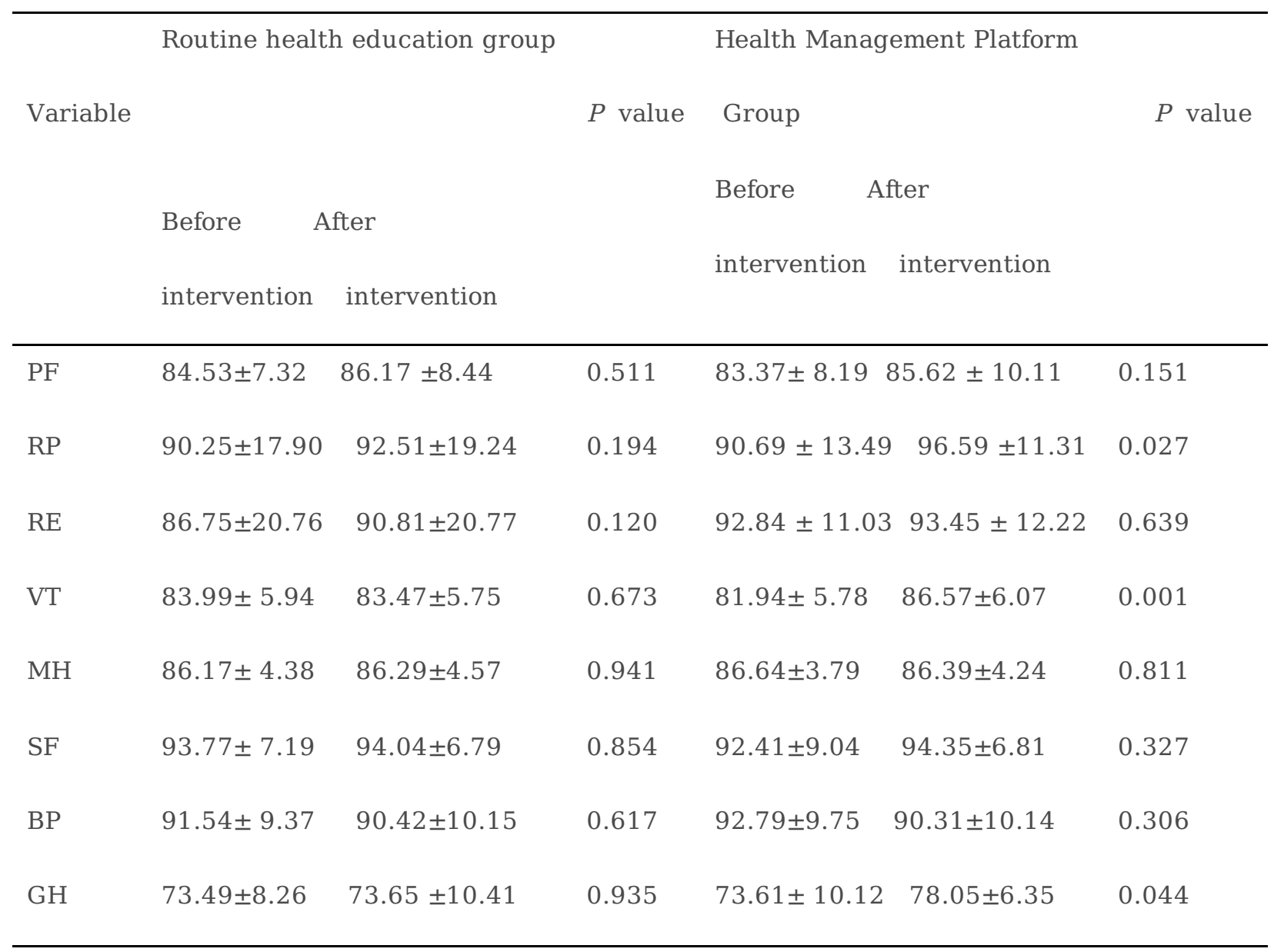

SF-36, Short Form Health Survey; PF, physical functioning; RP, role physical; BP, bodily pain; GH, general health; VT, vitality; SF, social functioning; RE, role emotional.

Table 5. Intergroup comparison of index differences (D) before and after the intervention 


\begin{tabular}{|c|c|c|c|c|}
\hline Variable & \multicolumn{2}{|c|}{$\begin{array}{l}\text { D-value of the routine health education } \\
\text { group }\end{array}$} & \multirow{2}{*}{$\begin{array}{l}\text { D-value of the health management platform } \\
\text { group } \\
\qquad-3.80 \pm 3.11\end{array}$} & \multirow{2}{*}{$\begin{array}{c}P \\
\text { value } \\
0.047^{*}\end{array}$} \\
\hline Weight (l & & $-0.12 \pm 3.42$ & & \\
\hline $\mathrm{WC}(\mathrm{cm})$ & & $-1.52 \pm 5.32$ & $-1.27 \pm 4.56$ & 0.237 \\
\hline HIPS $(\mathrm{cm}$ & & $-1.11 \pm 5.9$ & $-1.13 \pm 4.51$ & 0.918 \\
\hline WHR & & $0.01 \pm 0.08$ & $-0.01 \pm 0.07$ & 0.949 \\
\hline BMI & & $-0.14 \pm 1.68$ & $-1.73 \pm 1.20$ & $0.031^{*}$ \\
\hline SBP & & $1.58 \pm 4.17$ & $2.11 \pm 5.93$ & 0.684 \\
\hline DBP & & $3.68 \pm 2.15$ & $2.98 \pm 2.64$ & 0.734 \\
\hline ALT (U/L & & $-9.37 \pm 30.24$ & $-13.57 \pm 33.54$ & 0.833 \\
\hline AST (U/L & & $-4.67 \pm 11.58$ & $-6.52 \pm 17.12$ & 0.478 \\
\hline AST/ALT & & $0.04 \pm 0.11$ & $0.18 \pm 0.37$ & $0.037^{*}$ \\
\hline GGT & & $-1.51 \pm 3.34$ & $-0.92 \pm 2.41$ & 0.627 \\
\hline FPG & & $0.51 \pm 3.98$ & $0.52 \pm 1.01$ & 0.926 \\
\hline TGs & & $0.12 \pm 0.85$ & $-0.23 \pm 1.25$ & 0.337 \\
\hline CHOL & & $0.48 \pm 0.92$ & $0.17 \pm 1.56$ & 0.188 \\
\hline HDL-c & & $-0.04 \pm 0.54$ & $0.20 \pm 0.79$ & $0.046^{*}$ \\
\hline LDL-C & & $0.23 \pm 0.60$ & $0.04 \pm 0.37$ & 0.785 \\
\hline CAP $(\mathrm{dB} /$ & & $-4.13 \pm 7.45$ & $-48.42 \pm 10.13$ & $0.044^{*}$ \\
\hline Body fat & ontent (kg) & $-0.86 \pm 2.78$ & $-2.24 \pm 2.58$ & 0.194 \\
\hline Skeletal & nuscle content (kg) & $0.06 \pm 1.54$ & $0.25 \pm 1.92$ & 0.287 \\
\hline Visceral & at area $\left(\mathrm{cm}^{2}\right)$ & $-0.55 \pm 19.13$ & $-10.87 \pm 15.34$ & $0.047^{*}$ \\
\hline $\mathrm{PF}$ & & $1.52 \pm 9.57$ & $2.50 \pm 10.12$ & 0.446 \\
\hline $\mathrm{RP}$ & & $2.27 \pm 9.64$ & $5.47 \pm 10.41$ & 0.288 \\
\hline $\mathrm{RE}$ & & $4.04 \pm 12.84$ & $1.04 \pm 15.93$ & 0.442 \\
\hline VT & & $-0.45 \pm 5.37$ & $4.67 \pm 6.63$ & $0.001^{*}$ \\
\hline
\end{tabular}



$\mathrm{MH}$
$0.12 \pm 5.48$
$-0.04 \pm 4.78$
0.832
SF
$0.33 \pm 10.78$
$1.72 \pm 10.13$
0.753
BP
$-1.21 \pm 12.57$
$-2.50 \pm 13.58$
0.561
GH

Figures

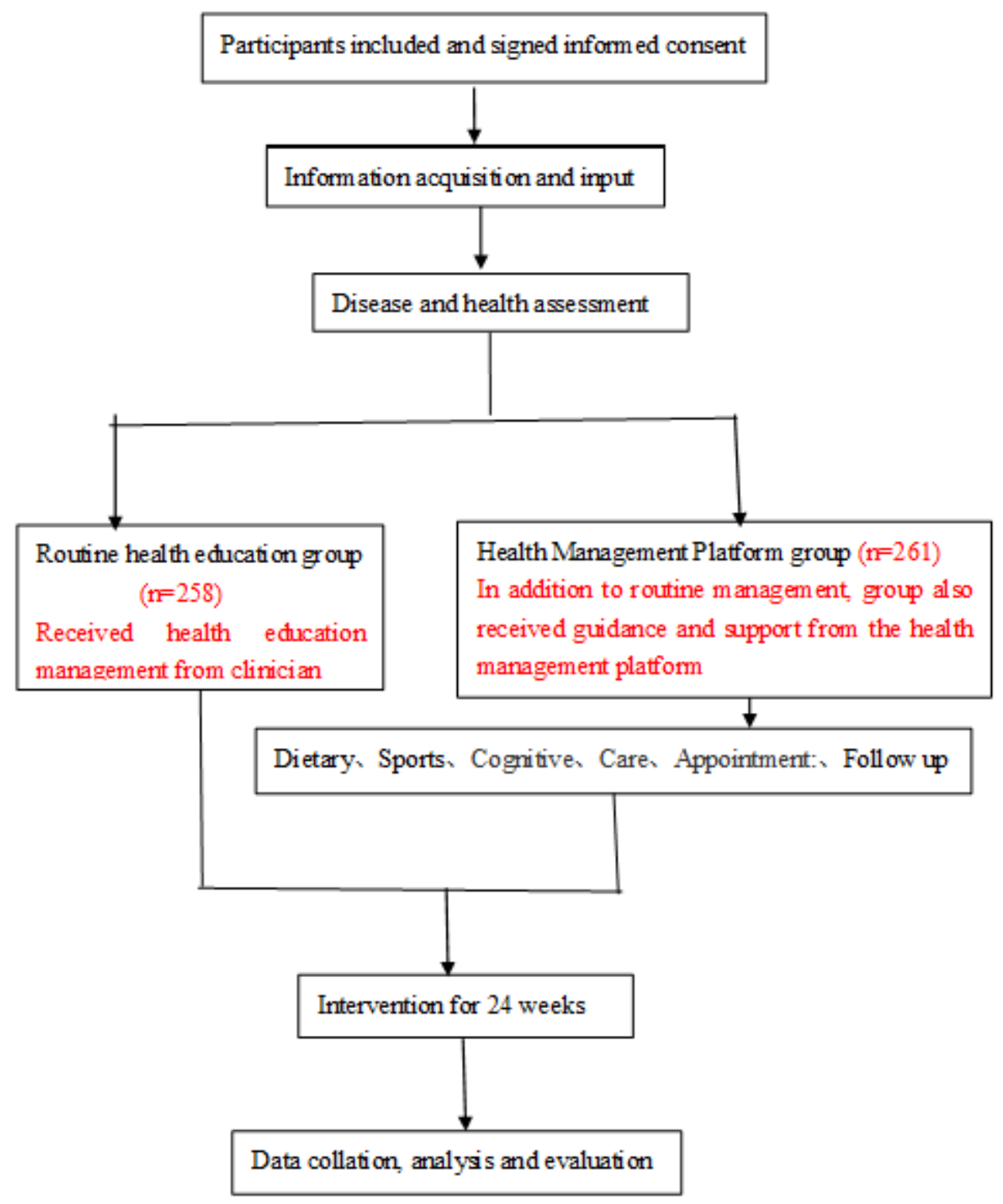

\section{Figure 1}

The technical roadmap. 


\section{Supplementary Files}

This is a list of supplementary files associated with this preprint. Click to download.

- CONSORT2010FlowDiagram.doc

- Equation.docx

- CONSORT2010Checklist.doc 